\title{
Hautpflege bei chronisch venöser Insuffizienz
}

\section{Was ist aus allergologischer Sicht zu berücksichtigen?}

\author{
J. Geier ${ }^{1}$, C. Erfurt-Berge ${ }^{2}$, V. Mahler ${ }^{3}$ \\ ${ }^{1}$ Informationsverbund Dermatologischer Kliniken (IVDK), Institut an der Universitätsmedizin Göttingen; \\ ${ }^{2}$ Hautklinik, Universitätsklinikum Erlangen; ${ }^{3}$ Abteilung Allergologie, Paul-Ehrlich-Institut, Langen
}

\begin{abstract}
Schlüsselwörter
Chronisch venöse Insuffizienz, Hautpflege, Kontaktallergie

\section{Zusammenfassung}

An Hand von Daten des Informationsverbundes Dermatologischer Kliniken (IVDK) der Jahre 2012-2016 wird das Spektrum von Kontaktsensibilisierungen bei 2011 Patienten mit Ulcus cruris venosum, Stauungsekzem und/oder einem Kontaktekzem bei chronisch venöser Insuffizienz (CVI) beschrieben, und mit dem Sensibilisierungsspektrum einer Kontrollgruppe $(n=30924)$ im ähnlichen Alter, aber ohne diese Erkrankungen verglichen. Unverändert zu früheren IVDK-Auswertungen zeigten CVI Patienten gehäuft Sensibilisierungen gegen das folgende Allergenspektrum: Perubalsam, Bestandteile von Salbengrundlagen, insbesondere Wollwachsalkohole, Antioxidantien, insbesondere tert-Butylhydrochi-
\end{abstract}

Korrespondenzadresse

Prof. Dr. med. Johannes Geier

Informationsverbund Dermatologischer Kliniken (IVDK)

Institut an der Universitätsmedizin Göttingen

Von-Bar-Str. 2-4

37075 Göttingen

Tel. 055150539625

Fax 055150539629

E-Mail: jgeier@gwdg.de non, Kolophonium, Duftstoffe und Parabene. Für die Hautpflege bei entsprechenden $\mathrm{Pa}$ tienten empfehlen sich daher duftstoff- und parabenfreie Produkte ohne Perubalsam (Myroxylon pereirae), Wollwachsalkohole (Lanolin alcohol) und tert-Butylhydrochinon (TBHQ). Dabei ist das sogenannte Parabenund Wollwachs-Paradox zu berücksichtigen: CVI Patienten können an ekzematös veränderter Haut am Unterschenkel auf Parabene oder Wollwachsalkohole reagieren, während diese Stoffe auf normaler Haut an anderen Körperregionen vertragen werden.

\section{Keywords}

Chronic venous insufficiency, skin care, contact allergy

Skin care in chronic venous insufficiency - What should be considered from an allergological point of view?

Phlebologie 2018; 47: 199-204

https://doi.org/10.12687/phleb2412-4-2018

Eingereicht: 14. Februar 2018

Angenommen: 29. Mai 2018

English version available at: www.thieme.de/phlebo

\section{Summary}

Based on data of the Information Network of Departments of Dermatology (IVDK) of the years 2012-2016, we describe the spectrum of contact sensitization in 2011 patients with venous ulcers, stasis dermatitis and/or chronic venous insufficiency (CVI) and compare it to the sensitization spectrum of an age-matched control group ( $n=30924)$ without these diseases. CVI patients frequently were sensitized to balsam of Peru, ointment base ingredients, particularly lanolin alcohols, antioxidants, in particular tert-butylhydroquinone, colophony, fragrances, and parabens. Compared to earlier IVDK data analyses, the sensitization spectrum is qualitatively unchanged. Skin care products for CVI patients should not contain Balsam of Peru (Myroxylon pereirae), fragrances, lanolin alcohols, tert-butylhydroquinone (TBHQ), and parabens. It should be noted that there are the so-called paraben and lanolin paradoxes: CVI patients may have intolerance reactions to parabens or lanolin alcohols on eczematous skin at the lower leg, while at the same time tolerating these compounds on healthy, normal skin in other regions of the body.

\section{Einleitung}

Wenngleich Hautpflegeprodukte im Allgemeinen sicher nicht zu denjenigen Dingen gehören, durch die besonders häufig epidermale Sensibilisierungen erworben werden, so ist - insbesondere im Bereich entzündlich veränderter Haut bei chronisch venöser Insuffizienz (CVI) - aufgrund der gestörten epidermalen Barriere und/oder spezieller immunologischer Bedingungen mit einem erhöhten diesbezüglichen Risiko zu rechnen $(1,2)$. Potentielle Allergene in entsprechenden Produkten können Konservierungsmittel, Duftstoffe, Bestandteile von Salbengrundlagen, Emulgatoren oder weitere Zusatzstoffe sein $(3,4)$.

Wir wollen an Hand von Daten des Informationsverbundes Dermatologischer Kliniken (IVDK) das Sensibilisierungs- spektrum von Patienten mit CVI darstellen, auf Besonderheiten hinweisen, und daraus Hinweise für die tägliche Praxis ableiten.

\section{Material und Methoden}

Der IVDK ist ein Zusammenschluss von derzeit 56 dermatologischen Kliniken in 
Deutschland, der Schweiz und Österreich, der die klinische Epidemiologie der Kontaktallergie untersucht. In den teilnehmenden Zentren werden (nach Aufklärung und Zustimmung) von allen epikutan getesteten Patienten anamnestische und klinische Daten sowie die Ergebnisse der Epikutantestung in lokalen Datenbanken gespeichert. In halbjährlichen Abständen werden diese Daten in pseudonymisierter Form an die Zentrale des IVDK übermittelt, die als An-Institut an der Universitätsmedizin Göttingen angesiedelt ist. Dort werden die Daten einer Qualitätskontrolle unterzogen und der zentralen Datenbank des IVDK hinzugefügt. Der IVDK verfügt über die weltweit größte Datenbank dieser Art, mit mehr Datensätzen von ca. 275000 Patienten (5).

Alle IVDK-Mitglieder führen die Epikutantestungen nach der Leitlinie der Deutschen Kontaktallergie-Gruppe (DKG) durch (6). Für die vorliegende Datenauswertung wurden die Testreaktionen an Tag 3 herangezogen. In wenigen Fällen lag keine Ablesung an Tag 3, dafür aber an Tag 4 vor, die dann für die Auswertung verwendet wurde. Als positiv wurden alle Reaktionen mit Erythem, Infiltrat, Papeln und ggf. Vesikeln gewertet, die mit +, ++, oder +++ codiert wurden. Die Testzubereitungen wurden von SmartPractice Europe bezogen. Für die Testungen wurden in mehr als 95\% der Fälle Finn-Chambers-on-Scanpor verwendet.

Insgesamt wurden in den Jahren 2012 bis 2016 in den dem IVDK angeschlossenen Kliniken 60.995 Patienten epikutan getestet. Von diesen litten 2.011 Patienten an Ulcus cruris venosum, Stauungsekzem und/oder einem Kontaktekzem bei CVI. Diese Patienten stellten die Untersuchungsgruppe („CVI-Patienten“) dar. Um ein spezielles Sensibilisierungsspektrum herausarbeiten zu können, wurde zu Vergleichszwecken eine Kontrollgruppe definiert. Da 90\% der Patienten der Untersuchungsgruppe 49-89 Jahre alt waren, und das Alter erhebliche Auswirkungen auf das zu beobachtende Sensibilisierungsspektrum hat, wurden als Kontrollgruppe Patienten derselben Altersgruppe ohne Ulcus cruris, Stauungsdermatitis oder Ekzem bei CVI gewählt, die im IVDK 2012 bis 2016 wegen eines allergischen Kontaktekzems

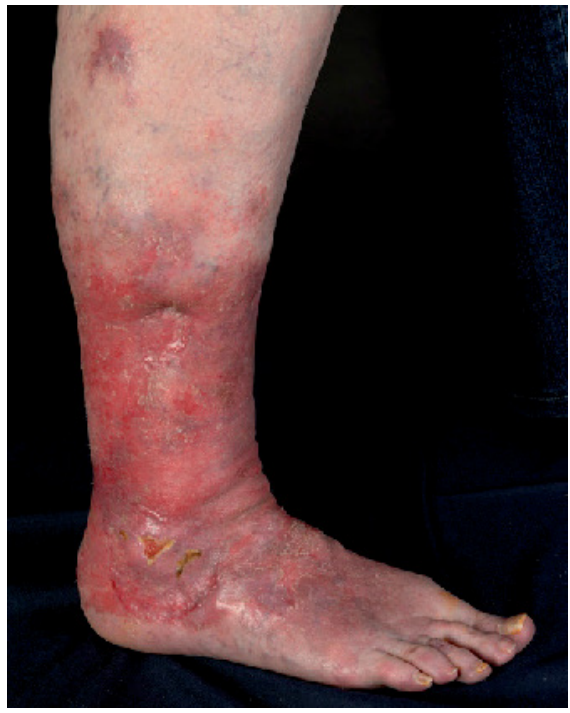

Abb. 1

Allergisches Kontaktekzem bei Ulcus cruris (Aufnahme Hautklinik Universitätsklinikum Erlangen).

oder zum Ausschluss einer Kontaktallergie bei anderen dermatologischen Erkrankungen epikutan getestet wurden. Dies waren 30.924 Patienten.

Zur statistischen Absicherung der Unterschiede in den Häufigkeiten positiver Reaktionen auf bestimmte Allergene in den beiden Gruppen wurden die exakten 95\%-Konfidenzintervalle (95\%-KIs) der Reaktionsquoten berechnet. Bei Nichtüberlappung der 95\%-KIs ist von einer statistischen Signifikanz auf 5\%-Niveau auszugehen.

\section{Ergebnisse}

Die Untersuchungsgruppe (2011 CVI-Patienten) bestand zu 59\% aus Frauen; der Altersdurchschnitt lag bei 72,4 \pm 12 Jahren, der Median bei 75 Jahren. In der Kontrollgruppe (30924 Patienten im Alter von 49-89 Jahren ohne CVI) waren 66\% Frauen; der Altersdurchschnitt lag bei $62,4 \pm 9,5$ Jahren, der Median bei 61 Jahren.

Es wurden die folgenden Testreihen der DKG getestet: Standardreihe (bei 1985 Patienten $=99 \%$ von 2.011), Externa-Inhaltsstoffe (1804 Pat. $=90 \%)$, Konservierungsmittel in Externa (1399 Pat. $=70 \%)$. Alle weiteren DKG-Testreihen wurden bei weniger als 30\% der CVI-Patienten getestet. Insgesamt zeigten 991 Patienten (49\%) mindestens eine positive Reaktion auf ein Allergen aus den DKG-Testreihen. In der Kontrollgruppe lag diese Quote bei 13607 von 30924 Patienten (44,0\%).

In Tabelle 1 sind die 25 in der Untersuchungsgruppe am häufigsten positiv getesteten Allergene mit ihren Reaktionsquoten aufgeführt. Zum Vergleich sind die entsprechenden Reaktionsquoten in der Kontrollgruppe angegeben. In dieser Tabelle sind ausschließlich solche Allergene berücksichtigt, die bei mindestens $85 \%$ der CVI-Patienten getestet wurden, da bei einem geringeren Anteil von einer selektiven Testung auszugehen ist, bei der die mehr oder weniger streng gestellte Indikation zur Testung die prozentuale Reaktionshäufigkeit so entscheidend beeinflusst, dass ein statistischer Vergleich nicht sinnvoll ist. Aufgrund dieser Beschränkung sind die Reaktionen auf Methylisothiazolinon (MI) 0,05\% Aqu. nicht enthalten, das zwar 3,3\% positive Reaktionen auslöste (95\%-KI: 2,5\%-4,3\%), aber bei nur $83 \%$ der Patienten getestet wurde. In der Kontrollgruppe wurde MI bei $73 \%$ der Patienten getestet, von denen $6,1 \%(5,8 \%-6,5 \%)$ positiv reagierten.

Einundzwanzig der 25 häufigsten Allergene wurden in beiden Gruppen bei mehr als 85\% der Patienten getestet, sodass ein Vergleich sinnvoll möglich ist. Auf 10 dieser Allergene ergaben sich signifikant erhöhte Reaktionsquoten in der Untersuchungsgruppe. Dabei handelte es sich um Duftstoffe und Bestandteile von Salbengrundlagen sowie Kolophonium. Die deutlichsten Unterschiede wurden bei tert-Butylhydrochinon (10,7\% vs. $1,6 \%)$, Amerchol L-101 (8,1\% vs. 3,1\%), Wollwachsalkoholen $(7,2 \%$ vs. $2,0 \%)$ und Cetylstearylalkohol (4,0\% vs. $0,7 \%)$ beobachtet. Bei 7 Testzubereitungen unterschieden sich die Reaktionsquoten nicht signifikant: Duftstoff-Mix, Propolis, Methyldibromo Glutaronitril, Kaliumdichromat, Ylang-Ylang Öl, Thiuram-Mix und Terpentin. Auf 4 Allergene reagierten die CVI-Patienten signifikant seltener als die Kontrollgruppe, nämlich auf Nickelsulfat, Chlormethylisothiazolinon / Methylisothiazolinon (MCI/ MI), Kobaltchlorid, und Hydroxyisohexyl 3-cyclohexene carboxaldehyde (HICC). Vier Allergene aus dem Bereich Salbengrundlagen wurden in der Kontrollgruppe 
Tab. 1

Reaktionsquoten auf die 25 am häufigsten positiv getesteten Allergene in der Untersuchungsgruppe. Aqu. $=$ Wasser (Aqua); $\mathrm{CVI}=$ chronisch venöse Insuffizienz; Vas. = Vaseline; $95 \%-K I=95 \%$-Konfidenzintervall; *Allergen wurde in der Kontrollgruppe nur selektiv getestet, weshalb ein Vergleich der Reaktionsquoten nicht sinnvoll ist.

\begin{tabular}{|c|c|c|c|}
\hline Allergen & $\begin{array}{l}\text { Testkonzentra- } \\
\text { tion, } \\
\text { Vehikel }\end{array}$ & $\begin{array}{l}\text { Untersuchungsgruppe } \\
\text { (2.011 CVI-Patienten) } \\
\text { Prozent positive Reaktionen } \\
{[95 \%-\mathrm{KI}]}\end{array}$ & $\begin{array}{l}\text { Kontrollgruppe } \\
\text { (30.924 Patienten ohne CVI) } \\
\text { Prozent positive Reaktionen } \\
{[95 \%-\mathrm{KI}]}\end{array}$ \\
\hline Perubalsam & $25 \%$ Vas. & $13,6[12,1-15,2]$ & $9,8[9,4-10,1]$ \\
\hline Duftstoff-Mix & $8 \%$ Vas. & $11,7[10,3-13,2]$ & $10,9[10,5-11,3]$ \\
\hline tert-Butylhydrochinon & $1 \%$ Vas. & $10,7[9,3-12,2]$ & $1,6[1,4-1,8]$ \\
\hline Duftstoff-Mix II & $14 \%$ Vas. & $8,0[6,9-9,3]$ & $5,9[5,6-6,2]$ \\
\hline Amerchol L-101 & $50 \%$ Vas & $8,1[6,8-9,4]$ & $3,1[2,9-3,4]$ \\
\hline Wollwachsalkohole & $30 \%$ Vas. & $7,2[6,1-8,5]$ & $2,0[1,8-2,2]$ \\
\hline Kolophonium & $20 \%$ Vas. & $5,3[4,3-6,4]$ & $3,9[3,7-4,2]$ \\
\hline Nickelsulfat & $5 \%$ Vas. & $5,3[4,4-6,4]$ & $13,4[13,0-13,8]$ \\
\hline Propolis & $10 \%$ Vas. & $4,2[3,3-5,1]$ & $4,4[4,2-4,7]$ \\
\hline Cetylstearylalkohol & $20 \%$ Vas. & $4,0[3,1-4,9]$ & $0,7[0,6-0,8]$ \\
\hline Propylenglykol* & $20 \%$ Aqu. & $4,1[3,2-5,1]$ & $1,2[1,1-1,4]$ \\
\hline Methyldibromo Glutaronitril & $0,2 \%$ Vas. & $3,2[2,4-4,1]$ & $2,9[2,7-3,1]$ \\
\hline Jasmin absolut & $5 \%$ Vas. & $3,3[2,5-4,2]$ & $1,5[1,4-1,7]$ \\
\hline Cocamidopropylbetain* & $1 \%$ Aqu. & $3,6[2,8-4,5]$ & $2,4[2,2-2,7]$ \\
\hline Kaliumdichromat & $0,5 \%$ Vas. & $3,2[2,5-4,1]$ & $4,0[3,7-4,2]$ \\
\hline Ylang-Ylang (I+II) Öl & $10 \%$ Vas. & $3,1[2,4-4,0]$ & $2,8[2,6-3,0]$ \\
\hline $\begin{array}{l}\text { Chlormethylisothiazolinon / Me- } \\
\text { thylisothiazolinon (MCl/MI) }\end{array}$ & $0,01 \%$ Aqu. & $2,8[2,1-3,7]$ & $5,1[4,8-5,3]$ \\
\hline Thiuram-Mix & $1 \%$ Vas. & $2,3[1,6-3,0]$ & $2,3[2,1-2,5]$ \\
\hline Kobaltchlorid & $1 \%$ Vas. & $2,2[1,6-3,0]$ & $4,3[4,0-4,5]$ \\
\hline Octylgallat* & $0,3 \%$ Vas. & $2,4[1,7-3,2]$ & $2,3[2,0-2,5]$ \\
\hline Sandelholzöl & $10 \%$ Vas. & $2,0[1,4-2,7]$ & $1,2[1,1-1,3]$ \\
\hline Butylhydroxyanisol (BHA)* & $2 \%$ Vas. & $1,9[1,3-2,6]$ & $0,3[0,2-0,4]$ \\
\hline Paraben-Mix & $16 \%$ Vas. & $1,5[1,0-2,2]$ & $0,6[0,5-0,7]$ \\
\hline Terpentin & $10 \%$ Vas. & $1,5[1,0-2,1]$ & $1,2[1,0-1,3]$ \\
\hline $\begin{array}{l}\text { Hydroxyisohexyl 3-cyclohexene } \\
\text { carboxaldehyde (HICC) }\end{array}$ & $5 \%$ Vas. & $1,4[0,9-2,0]$ & $2,3[2,1-2,5]$ \\
\hline
\end{tabular}

nur selektiv, also mehr oder weniger gezielt, getestet. Bei 3 dieser 4 Allergene ergaben sich dennoch höhere Reaktionsquoten in der Untersuchungsgruppe: Propylenglykol (4,1\% vs. 1,4\%), Cocamidopropylbetain (3,6\% vs. $2,4 \%)$, und Butylhydroxyanisol $(1,9 \%$ vs. $0,3 \%)$.

\section{Diskussion}

Eine kürzlich publizierte IVDK-Datenanalyse zeigte, dass die Häufigkeit des allergischen Kontaktekzems bei Patienten mit Ulcus cruris venosum, und Stauungsekzem bei CVI in den Jahren 2004-2013 im Ver- gleich zum Zeitraum 1994-2003 signifikant von 25,9\% auf 16,9\% zurückgegangen ist, das Allergenspektrum aber im Wesentlichen unverändert war (4). Auch in der hier vorgestellten neueren Datenauswertung fanden wir ein qualitativ unverändertes Allergenspektrum (bei allerdings teilweise überlappenden Auswertungszeiträumen). Die Intention der vorliegenden Arbeit ist nicht, die unter epidemiologischen Aspekten differenziert und detailliert analysierten Daten der genannten Publikation zu reproduzieren, sondern das aktuell vorgefundene Allergenspektrum darzustellen und unter dem Aspekt der Bedeutung für die Hautpflege bei entsprechenden Patienten $\mathrm{zu}$ bewerten.

\section{Perubalsam}

Perubalsam führte die „Hitliste“ der häufigsten Allergene an; entsprechende Sensibilisierungen traten signifikant häufiger auf als in der Kontrollgruppe. Perubalsam (INCI: Myroxylon pereirae), das Wundsekret des im Mittelamerika wachsenden Perubalsam-Baumes, wird wegen seiner granulations- und wundheilungsfördernden Wirkung geschätzt, hat aber ein erhebliches sensibilisierendes Potenzial. Der Perubalsam enthält über 250 verschiedene 
Komponenten, von denen mindestens 20 als Kontaktallergen bekannt sind, u.a. Zimtalkohol, Eugenol und Isoeugenol, die auch im Duftstoff-Mix enthalten sind. Fünfzehn Bestandteile des Perubalsams wurden auch in Propolis gefunden, u.a. Benzoesäure, Benzylalkohol, Benzylcinnamat, Coniferylbenzoat, Farnesol, Vanillin und Zimtsäure, wodurch sich erklärt, warum viele Patienten gleichzeitig auf Perubalsam und Propolis allergisch reagieren. Nicht alle bekanntermaßen sensibilisierende Inhaltsstoffe des Perubalsams stehen als eigene Testsubstanzen zur Verfügung (7, 8).

\section{Bestandteile von Salbengrundla-} gen

Bemerkenswert ist die hohe Quote allergischer Reaktionen auf tert-Butylhydrochinon (INCI: TBHQ), das als Antioxidans in Kosmetika und Hautpflegeprodukten in Konzentrationen von $0,1-1 \%$ eingesetzt wird. Der Stoff ist auch für Lebensmittel zugelassen (Zusatzstoff Nr. E 319). Im Vergleich zu Daten aus den Jahren aus den Jahren 1994/1995 ist die Sensibilisierungsquote bei CVI-Patienten leicht angestiegen (3). Ob dies auf den vermehrten Einsatz von tert-Butylhydrochinon in Hautpflegeprodukten zurückgeht, entzieht sich unserer Kenntnis.

Wollwachsalkohole (INCI: Lanolin alcohol) sind ein Gemisch aus organischen Alkoholen, die aus Wollfett (Lanolin) gewonnen werden. Amerchol L101 ist ein Handelsprodukt, das neben Wollwachsalkoholen auch Paraffinum liquidum enthält (9). Wollwachsalkohole, die als Salbengrundlage und Emulgator fungieren, können zweifelsohne sensibilisieren. Ob allerdings jede positive Reaktion auf die Testzubereitung Wollwachsalkohole 30\% Vas. oder - noch mehr - auf Amerchol L 101 $50 \%$ Vas. auch wirklich eine Sensibilisierung anzeigt, ist fraglich, da diese Testzubereitungen die Haut leicht irritieren können und dann auch zu falsch-positiven Reaktionen führen können (10). Zudem gibt es große Unterschiede zwischen den verschiedenen Wollwachsalkohol-Gemischen, so dass es vorkommen kann, dass ein $\mathrm{Pa}$ tient im Epikutantest positiv reagiert, aber sein verwendetes (wollwachsalkoholhalti- ges) Produkt verträgt oder umgekehrt (11). Schließlich kommt es vor, dass Patienten im Bereich ekzematös vorgeschädigter Haut, wie z.B. bei der Stauungsdermatitis, allergisch auf wollwachsalkoholhaltige Externa reagieren, während sie sie auf intakter Haut an anderen Körperbereichen reaktionslos tolerieren - ein Phänomen, dass unter der Bezeichnung "Lanolin Paradox" Eingang in die wissenschaftliche Literatur gefunden hat (12).

Auch bei Cetylstearylalkohol (INCI: Cetearyl alcohol) handelt es sich um ein als Salbengrundlage bzw. Emulgator eingesetztes Gemisch, nämlich aus Cetylalkohol (Hexadecanol) und Stearylalkohol (Octadecanol). Die Testzubereitung ist deutlich weniger irritierend und daher diagnostisch zuverlässiger. Im Allgemeinen ein seltenes Allergen, spielt es bei CVI-Patienten offenbar doch eine bedeutendere Rolle als Sensibilisator $(3,4,9)$.

Propylenglykol (INCI: Propylene glycol) führte in der Untersuchungsgruppe deutlich häufiger zu positiven Reaktionen als in der Kontrollgruppe, obwohl die Substanz dort mutmaßlich gezielter getestet wurde. Die von der DKG verwendete Testzubereitung Propylenglykol 20\% Aqu. hat ein gewisses irritatives Potential, so dass falsch positive Reaktionen auftreten können (10). Es wäre allerdings nicht ohne weiteres einsichtig, warum dies bei CVI-Patienten häufiger der Fall sein sollte als bei anderen $\mathrm{Pa}$ tienten. Insofern ist davon auszugehen, dass CVI-Patienten tatsächlich häufiger gegen Propylenglykol, das in Externa zur Viskositätseinstellung und zum Feuchthalten eingesetzt wird, sensibilisiert sind.

Während das Antioxidans Octylgallat (INCI: Ethylhexyl gallate) bei CVI-Patienten nicht wesentlich häufiger zu sensibilisieren scheint als in der Kontrollgruppe, ist ein solche Tendenz bei Butylhydroxyanisol (INCI: BHA), das ebenfalls als Antioxidans eingesetzt wird, in unseren Daten erkennbar. Auch auf BHA lag die Reaktionsquote in der Untersuchungsgruppe erheblich höher als in der Kontrollgruppe, obwohl dort wahrscheinlich gezielter getestet wurde. Die Testzubereitung Octylgallat 0,3\% Vas. ist - wie Propylenglykol 20\% Aqu. - als „Problemallergen“ mit vielen fraglichen, irritativen oder falsch positiven Reaktionen behaftet (10), was bei BHA 2\% Vas. nicht der Fall ist.

\section{Kolophonium}

Chemisch modifiziertes Kolophonium ist unter anderem in Hydrokolloidverbänden enthalten, die auf chronischen Wunden, Ulcera etc. zum Einsatz kommen $(4,13)$. Hier liegt wahrscheinlich eine bedeutende Allergenquelle für die Sensibilisierung gegen Kolophonium bei CVI-Patienten, die im Vergleich zur Kontrollgruppe mit leicht erhöhter Häufigkeit festzustellen war. Man muss allerdings davon ausgehen, dass eine gewisse Dunkelziffer besteht, weil mit der standardisierten Testsubstanz Kolophonium nicht alle Sensibilisierungen auch gegen chemisch modifiziertes Kolophonium diagnostiziert werden. In einer früheren Untersuchung reagierten ca. 19\% der Patienten mit Kontaktallergie gegen modifiziertes Kolophonium nicht auf die Standard-Testsubstanz (13).

\section{Parabene}

In der Epikutantest-Standardreihe der DKG ist ein Paraben-Mix (16\% Vas.) enthalten, der vier Parabene in Konzentrationen enthält, die weit über den Höchstkonzentrationen liegen, die nach EU-Kosmetikverordnung (14) für Kosmetika und Körperpflegeprodukte zugelassen sind. Offenbar führt diese hohe Konzentration $\mathrm{zu}$ Irritationen, denn nähere Analysen ergaben, dass bis zu 50\% der positiven Reaktionen falsch positiv und schlecht reproduzierbar sind (15). Zieht man dies von den ohnehin niedrigen Reaktionsquoten ab, so muss man feststellen, dass Parabene keine bedeutenden Kontaktallergene sind. Gleichwohl können insbesondere bei $\mathrm{Pa}$ tienten mit Stauungsdermatitis klinisch relevante Paraben-Allergien auftreten. Fisher beschrieb 1973 das so genannte „ParabenParadox“: Viele Patienten reagieren im Bereich ekzematös vorgeschädigter Haut, wie z.B. bei der Stauungsdermatitis, allergisch auf parabenhaltige Externa, während sie auf intakter Haut an anderen Körperbereichen Parabene reaktionslos tolerieren (16). Die klinische Erfahrung bestätigt diese Beobachtung. 


\section{Weitere Allergene}

Unter den häufigsten Allergenen in der Untersuchungsgruppe sind auch etliche Duftstoffe, wobei im Vergleich zur Kontrollgruppe nur zum Teil erhöhte Reaktionsquoten beobachtet wurden. Bemerkenswerter Weise fanden sich jedoch über den Paraben-Mix hinaus keine erhöhten Quoten an Sensibilisierungen gegen Konservierungsmittel wie z.B. Methyldibromo Glutaronitril, MCI/MI oder MI.

\section{Schlussfolgerung für die Praxis}

Patienten mit Ulcus cruris venosum und/ oder Stauungsdermatitis bei CVI haben gehäuft Sensibilisierungen gegen Perubalsam, Salbengrundlagen, insbesondere Wollwachsalkohole, Antioxidantien, insbesondere tert-Butylhydrochinon, Kolophonium, Duftstoffe und Parabene. Für die Hautpflege dieser speziellen Patientengruppe empfehlen sich daher duftstoffund parabenfreie Produkte ohne Perubalsam (Myroxylon pereirae), Wollwachsalkohole (Lanolin alcohol) und tert-Butylhydrochinon (TBHQ).

Vor dem Hintergrund des hier dargestellten (und seit langem bekannten) Sensibilisierungsspektrums ist es unverständlich, warum zum Beispiel ein zur Hautpflege angepriesenes Produkt wie Peru-Lenicet $^{\natural}$, das mit Perubalsam, Lanolin und Kolophonium gleich drei der häufigsten Allergene von CVI-Patienten enthält, noch immer auf dem Markt ist.

\section{Danksagung}

Folgende Zentren des IVDK trugen Daten zu der Analyse bei (in alphabetischer Reihenfolge): Aarau (J. Grabbe), Augsburg (A. Ludwig), Basel (A. Bircher), Berlin Charité (M. Worm), Bern (D. Simon), Bielefeld (I. Effendy), Bochum (H. Dickel), Bremen (D. Meyersburg, N. Patsinakidis), Dessau (A. Jung, U. Lippert), Detmold (St. Nestoris), Dortmund (P.J. Frosch, B. Mydlach, K. Kügler, U. Beiteke), Dresden (P. SpornraftRagaller, A. Bauer), Dresden Friedrichstadt (A. Koch), Erlangen (V. Mahler), Essen (U. Hillen), Falkenstein (D. Vieluf), Freuden- berg (Ch. Szliska), Gera (H. Grunwald-Delitz, M. Kaatz), Graz (B. Kränke, W. Aberer), Greifswald (M. Jünger), Göttingen (Th. Fuchs, J. Geier, T. Buhl), Halle (B. Kreft), Hamburg Dermatologikum (V. Martin, K. Reich, K. Breuer, D. Vieluf), Hamburg UKE (E. Coors, J. Witte), Hannover (Th. Werfel, A. Kapp), Heidelberg (K. Schäkel), Heilbronn (H. Löffler), Homburg / Saar (C. Pföhler), Jena (S. Schliemann), Lausanne CHU (P. Spring, C. Curdin), Lausanne CEDDECC (P. Spring), Leipzig (R. Treudler), Lübeck (I. Shimanovich, U. Jappe, K. Hartmann, A. Recke), Mainz (D. Becker), Mannheim (W. Ludwig-Peitsch, A. Schmieder), Marburg (M. Hertl, W. Pfützner), Minden (J. Hoffmann, R. Stadler), München LMU (B. Przybilla, P. Thomas, S. Molin), München Schwabing (M. Agathos, K. Ramrath, G. Isbary), München TU (U. Darsow, T. Biedermann), Münster (B. Hellweg, R. Brehler, M. Behring), Nürnberg (A. Bachtler, K. Ertner, V. Baur), Oldenburg (M. Padeken, O. Kautz), Osnabrück (Ch. Skudlik, S.M. John), Rostock (J. Trcka), Stuttgart (J. RiekerSchwienbacher), Tübingen (T. Biedermann, J. Fischer), Ulm (J. Weiss), Würzburg (A. Trautmann), Zwickau (D. Teubner, D. Mechtel), Zürich (B. Ballmer-Weber, A. Navarini).

\section{Hinweis}

Teile dieser Datenanalyse wurden auf der 59. Jahrestagung der Deutschen Gesellschaft für Phlebologie, Stuttgart, 20.-23. Sep. 2017, vorgetragen.

\section{Interessenkonflikt}

J. Geier und V. Mahler haben keine Interessenkonflikte. C. Erfurt-Berge hat Reisekosten und Vortragshonorare von BSN Medical GmbH, Lohmann \& Rauscher $\mathrm{GmbH}$ \& Co.KG, Smith \& Nephew und Urgo $\mathrm{GmbH}$ erhalten.

\section{Ethische Richtlinien}

Für das Manuskript wurden keine Studien an Menschen oder Tieren durchgeführt.

\section{Literatur}

1. Bahmer F. Immunhistologische Charakterisierung stauungsdermatitisch veränderter Unterschenkelhaut. Zeitschrift für Hautkrankheiten 1987; 14: 1056-1063.

2. Weyl A, Vanscheidt W, Weiss JM, Peschen M, Schopf E, Simon J. Expression of the adhesion molecules ICAM-1, VCAM-1, and E-selectin and their ligands VLA-4 and LFA-1 in chronic venous leg ulcers. Journal of the American Academy of Dermatology 1996; 34: 418-423.

3. John SM, Geier J. Unterschenkelekzem bei chronisch-venöser Insuffizienz - Aktuelle allergologische Aspekte. Allergenspektrum bei „Beinpatienten“ im IVDK 1994-1995. Zeitschrift für Dermatologie 1998; 184: 16-23.

4. Erfurt-Berge C, Geier J, Mahler V. The current spectrum of contact sensitization in patients with chronic leg ulcers or stasis dermatitis-new data from the Information Network of Departments of Dermatology (IVDK). Contact Dermatitis 2017; 77: 151-158.

5. Schnuch A, Geier J, Lessmann H, Arnold R, Uter W. Surveillance of contact allergies: methods and results of the Information Network of Departments of Dermatology (IVDK). Allergy 2012; 67: 847-857.

6. Schnuch A, Aberer W, Agathos M, Becker D, Brasch J, Elsner P, Frosch PJ, Fuchs Th, Geier J, Hillen U, Löffler H, Mahler V, Richter G, Szliska C für die Deutsche Kontaktallergie-Gruppe. Durchführung des Epikutantests mit Kontaktallergenen. Leitlinien der Deutschen Dermatologischen Gesellschaft (DDG) und der Deutschen Gesellschaft für Allergie und klinische Immunologie (DGAKI). Journal der Deutschen Dermatologischen Gesellschaft 2008; 6: 770-775.

7. Hausen BM, Brinkmann J, Dohn W. Lexikon der Kontaktallergene. 3. Ergänzungslieferung, P9, P10 und K4. Landsberg: ecomed Verlag, 1996

8. Hausen BM. Contact allergy to balsam of peru. II. Patch test results in 102 patients with selected balsam of peru constituents. American Journal of Contact Dermatitis 2001; 12: 93-102.

9. de Groot AC. Monographs in Conact Allergy, Volume1, Part 1. Non-fragrance allergens in cosmetics. CRC Press, Boca Raton, FL, USA, 2018; 221-225, 698-710.

10. Geier J, Weisshaar E, Lessmann H, Becker D, Dickel H, Häberle M, John SM, Mahler V, Skudlik C, Wagner E, Wehrmann W, Werfel T, Zagrodnik F, Diepgen TL für die Arbeitsgruppe „Bewertung der Allergene bei BK 5101“ der Arbeitsgemeinschaft für Berufs- und Umweltdermatologie in der Deutschen Dermatologischen Gesellschaft. Bewertung von Epikutantestreaktionen auf „Problemallergene" mit vermehrt fraglichen oder schwach positiven Reaktionen. Dermatologie in Beruf und Umwelt 2010; 58: 34-38.

11. Lee B, Warshaw E. Lanolin allergy: history, epidemiology, responsible allergens, and management. Der,atitis 2008; 19: 63-72.

12. Wolf R. The lanolin paradox. Dermatology 1996; 192: 198-202.

13. Geier J, Hausen BM. Epikutantestung mit chemisch modifiziertem Kolophonium. Ergebnisse einer Studie der Deutschen Kontaktallergie- 
Gruppe (DKG) und des Informationsverbundes Dermatologischer Kliniken (IVDK). Dermatologie in Beruf und Umwelt 2000; 48: 43-47.

14. Verordnung (EG) Nr. 1223/2009 des Europäischen Parlaments und des Rates vom 30. November 2009 über kosmetische Mittel. Amtsblatt der Europäischen Union L 342/59-208 vom 22.12.2009.
Letzte Änderung: Verordnung (EU) 2017/238 der Kommission vom 10. Februar 2017 zur Änderung von Anhang VI der Verordnung (EG) Nr. 1223/2009 des Europäischen Parlaments und des Rates über kosmetische Mittel. Amtsblatt der Europäischen Union L36/37-38 vom 11.2.2017.
15. Uter W, Schnuch A, Geier J, Agathos M. Epikutantest-Reaktionen auf Paraben-Mixe und ihre Aufschlüsselungen. Studien der DKG und des IVDK, 1990-2000. Allergologie 2002; 25: 194-202. 16. Fisher AA. The paraben paradox. Cutis 1973; 12 : 830-832. 